\title{
CÂNCER INFANTIL: UMA PROPOSTA DE AVALIAÇÃO DAS ESTRATÉGIAS DE ENFRENTAMENTO DA HOSPITALIZAÇÃO
}

\author{
CHILDHOOD CANCER: AN ASSESSMENT OF \\ HOSPITALIZATION COPING STRATEGIES PROPOSAL
}

\author{
Alessandra Brunoro MOTTA ${ }^{1}$ \\ Sônia Regina Fiorim ENUMO²
}

\begin{abstract}
RESUMO
A criança com câncer precisa se adaptar à hospitalização, utilizando estratégias de enfrentamento adequadas a fim de minimizar os efeitos negativos. Visando à elaboração de um instrumento de avaliação das estratégias de enfrentamento da hospitalização, 28 crianças, de 6 a 12 anos, inscritas no serviço de oncologia de um hospital público de Vitória, ES, foram submetidas à aplicação do instrumento: Avaliação das Estratégias de Enfrentamento da Hospitalização, com 41 pranchas ilustradas, divididas nos conjuntos: A: Enfrentamento da hospitalização, e B: Brincar no hospital. Os resultados referentes à adequação do instrumento mostraram um percentual de $88,4 \%$ e $94,6 \%$ de adequação das pranchas do Conjunto $A$ e do Conjunto $B$, respectivamente. Os resultados das respostas ao instrumento indicaram um padrão de respostas de enfrentamento mais facilitador (brincar, conversar, tomar remédio e rezar) do que não-facilitador (esconder-se, brigar, sentir culpa, fazer chantagem). O instrumento mostrou-se adequado à compreensão e ao atendimento psicológico à criança hospitalizada.
\end{abstract}

Palavras-chave: estratégias de enfrentamento; hospitalização infantil; câncer infantil.

\section{ABSTRACT}

In order to diminish the negative effects of hospitalization, the child who has cancer needs to adapt herself/himself by using adequate coping strategies.

\footnotetext{
${ }^{1}$ Programa de Pós-Graduação em Psicologia, Universidade Federal do Espírito Santo. Av. Fernando Ferrari, s/n, Campus Universitário de Goiabeiras, Prédio CEMUNI VI, 29060-900, Vitória, ES, Brasil. Correspondência para/Correspondence to: A.B. MOTTA. E-mail: <abmotta.vix@zaz.com.br>.

2 Departamento de Psicologia Social e do Desenvolvimento e do Programa de Pós-Graduação em Psicologia, Universidade Federal do Espírito Santo, Vitória, ES, Brasil.
} 


\begin{abstract}
Focused on the design of an assessment tool for the hospitalization coping strategies, 28 children (6 to 12) registered on the Oncology Service from a public hospital in Vitória, Espírito Santo, were submitted to the following instruments: coping Hospitalization Assessment Instrument, with 41 pictures, divided by sets: Set A: Facing hospitalization and Set B: Playing in the hospital. The instrument adequacy results have shown a percentage of $88.4 \%$ e $94.6 \%$ of adequacy in the pictures from Set $A$ and Set B, respectively. The instrument responses results have revealed a larger tendency to the optimistic pattern of coping answers (play, talk, take medicine and pray), than to the non-optimistic (hide, fight, blame yourself, blackmail). The instrument was adequate to the psychological comprehension and attendance for the hospitalized children.
\end{abstract}

Key-words: coping strategies; children hospitalization; cancer in children.

O tratamento do câncer infantil tem como característica o fato de ser prolongado, demandando um tempo considerável de hospitalização e expondo a criança a procedimentos invasivos e desagradáveis, tanto física quanto emocionalmente. A criança precisa, então, adaptar-se a essa nova situação, sendo necessária a utilização de estratégias de enfrentamento adequadas.

A expressão estratégias de enfrentamento é tradução do termo coping, definido por Antoniazzi, Dell'Aglio e Bandeira (1998, p. 274) como: "(...) o conjunto de estratégias utilizadas pelas pessoas para adaptarem-se a circunstâncias adversas".

A perspectiva de coping adotada neste estudo refere-se à proposta da década de 1960, utilizada especialmente por Susan Folkman e Richard S. Lazarus, e encontra-se dentro de uma abordagem comportamental-cognitiva, que propõe um modelo de coping dividido em duas categorias funcionais: 1) o coping focalizado no problema - a situação a ser enfrentada é avaliada como suscetível à mudança, constituindo-se um esforço para atuar na situação que originou o stress e 2) o coping focalizado na emoção - a avaliação indica que nada pode ser feito para modificar a situação estressante; o esforço neste caso está voltado para a regulação do estado emocional (Lazarus \& Folkman, 1984).

Em revisão sobre o conceito e a metodologia de coping, Cerqueira (2000) afirma que o estudo do coping constitui-se uma área problemática, especialmente pela falta de clareza conceitual e por questões metodológicas presentes nos estudos que visam medi-lo. Dentre as dificuldades, destacam-se: construção de medidas restritas ao uso da análise fatorial explorativa decorrente da falta de direção que a teoria oferece; dificuldade em identificar que itens são válidos para representar diferentes estratégias (resolvida ao se elaborar itens diferentes para diferentes situações e populações); natureza do conceito de coping e dificuldades relacionadas ao aspecto psicométrico das medidas de coping (Cerqueira, 2000).

Analisando agora as pesquisas sobre coping em crianças, Antoniazzi et al. (1998) alertam para o pequeno número de pesquisadores que se dedicam a essa área, indicando a necessidade de elaboração e adequação de instrumentos dirigidos a crianças e adolescentes em condição de stress.

Procurou-se, então, identificar os principais estressores da hospitalização infantil: a doença; a dor; o ambiente hospitalar pouco familiar; a exposição a procedimentos médicos invasivos; a separação dos pais; o stress dos acompanhantes; a ruptura da rotina de vida e adaptação a uma nova rotina imposta e desconhecida; a perda da autonomia, controle e competência pessoal; a incerteza sobre a conduta mais apropriada; e a morte (Méndez, Ortigosa \& Pedroche, 1996). Essa identificação é fundamental, pois, como alertam Lazarus e Folkman (1984), há a 
necessidade de se conhecer o que a pessoa, de fato, está enfrentando, uma vez que, quanto mais definido estiver o contexto da situação a ser enfrentada, mais fácil será articular o ato ou pensamento particular de enfrentamento com a demanda da situação.

No caso da criança com câncer, todos os estressores citados anteriormente estão presentes, além de intensificados pelo tratamento prolongado.

Buscando atender as questões descritas anteriormente, a presente pesquisa teve como objetivo elaborar uma proposta de avaliação das estratégias de enfrentamento da hospitalização em crianças com câncer, buscando contribuir para o atendimento hospitalar e para o direcionamento de intervenções que tornem o suporte social mais adequado às instituições hospitalares.

\section{MÉTODO}

\section{Participantes}

Participaram deste estudo 28 crianças (9 meninas e 19 meninos), com idade entre 6 e 12 anos (média: 9 anos), inscritas no serviço de oncologia de um hospital infantil público de Vitória, Espírito Santo, vinculado ao Sistema Único de Saúde e considerado referência no Espírito Santo para o tratamento de câncer infantil, recebendo pacientes de todo o Estado, do sul da Bahia e do leste de Minas Gerais.

\section{Instrumentos}

Os instrumentos utilizados na coleta de dados foram os seguintes: a) Roteiro para registro de dados familiares e de saúde obtidos em prontuários médicos e fichas sociais a fim de levantar informações gerais para caracterização das crianças; e b) Instrumento de Avaliação de Estratégias de Enfrentamento da Hospitalização $(\mathrm{AEH})$, elaborado para a avaliação das estratégias de enfrentamento da hospitalização utilizadas pelas crianças com câncer.

\section{Procedimento}

Para alcançar o objetivo proposto, a pesquisa foi executada em quatro etapas, no período de junho a agosto de 2000:

Primeira etapa - identificação dos sujeitos: a partir do cadastro dos pacientes (nome, data de nascimento, fase do tratamento, data de inscrição no serviço de oncologia), foi possível selecionar os que se encontravam no intervalo de idade estabelecido (6 a 12 anos) e que estavam freqüentando o hospital regularmente, no mínimo, uma vez por mês.

Segunda etapa - caracterização dos sujeitos: levantamento documental nos prontuários médicos e fichas de dados sociais dos sujeitos, feito no ambulatório de oncologia, em dias e horários sem atendimento para não prejudicar a rotina do serviço.

Terceira etapa - elaboração do instrumento de avaliação das estratégias de enfrentamento. O instrumento proposto recebeu o nome de Instrumento de Avaliação das Estratégias de Enfrentamento da Hospitalização e teve como base para a sua elaboração outros instrumentos disponíveis sobre stress, ansiedade infantil e qualidade de vida: a Escala de Stress Infantil (ESI) (Lipp \& Luccarelli, 1998), o Inventário de Ansiedade Traço-Estado (IDATE-C) (Spielberg, 1983), o Autoquestionnaire Qualité de Vie Enfant Imagé (AUQEI), de Manificat e Dazord (Assumpção, Kuczynski, Sprovieri \& Aranha, 2000), a adaptação do Inventário de Estratégias de Coping de Folkman e Lazarus, feita por Savóia, Santana e Mejias (1996) e a Escala de Avaliação do Comportamento da Criança, proposto por Löhr e Silvares (Löhr, 1998).

Para uma maior adequação do instrumento e sugestão de temáticas a serem abordadas, foram feitas observações prévias do cotidiano da criança no ambulatório e na enfermaria do hospital, quando se registraram comportamentos indicativos de formas de enfrentar a situação (cantar, brincar, chorar, dormir, ler, entre outros).

$\mathrm{O}$ instrumento elaborado baseou-se, também, nos dados da pesquisa sobre conceitos 
de saúde, doença e morte, de Fávero e Salim (1995), no que se refere ao uso do desenho como instrumento de coleta de dados. Essa análise permitiu identificar que as crianças representavam a doença da seguinte forma: o doente com alterações corporais (magreza), com expressões faciais que denotam sentimentos de tristeza (boca desenhada com traços descendentes e a presença de lágrima). Essas características foram levadas em consideração, objetivando-se atingir certa coerência com as percepções das crianças e, assim, aumentar as possibilidades de adequação do instrumento.

Assim, foi proposto o AEH, composto por: 1) um roteiro de entrevista, com cinco perguntas a serem feitas para a criança sobre: a) suas estratégias de enfrentamento da hospitalização (pensamentos, sentimentos e atitudes); b) o que gostaria de fazer no hospital; e c) o brincar (definição e preferência do que e com quem brincar no hospital); 2) um caderno espiral de desenho, com 21 cenas desenhadas em preto e branco sobre temas que retratam possíveis estratégias de enfrentamento da hospitalização (Conjunto de Pranchas A: Enfrentamento da Hospitalização); 3) um caderno espiral de desenho, com 20 tipos de brincadeiras desenhadas em preto e branco (Conjunto de Pranchas
B: Brincar no Hospital); e 4) folhas de registro das respostas.

O Conjunto de Pranchas A: Enfrentamento da Hospitalização contém cenas que representam comportamentos descritos no Quadro 1.

Por meio do Conjunto de Pranchas A: Enfrentamento da Hospitalização", procurou-se conhecer as formas pelas quais a criança vem enfrentando a sua doença e o tratamento no hospital. As cenas desse conjunto foram selecionadas e distribuídas em duas categorias: respostas facilitadoras (F) e não-facilitadoras (NF). Entenda-se por respostas facilitadoras aquelas que se referem a uma estratégia considerada adaptativa, e por respostas não-facilitadoras as consideradas não adaptativas à situação, segundo avaliação prévia da pesquisadora, apoiada no senso comum de adequação.

Com as pranchas A1: Brincar, A4: Assistir TV, A7: Cantar e dançar, A10: Estudar, A14: Ouvir música e A17: Ler gibi, procurou-se abranger estratégias facilitadoras, características de atividades recreativas e/ou cotidianas da vida normal da criança. $O$ uso de tais estratégias para o enfrentamento de uma situação adversa, no caso a hospitalização, encontra apoio no item 3 (Procurei trabalhar ou fazer alguma atividade

Quadro 1. Conjunto de Pranchas A: Enfrentamento da Hospitalização.

\begin{tabular}{|c|c|c|c|c|c|c|c|}
\hline Prancha & Conteúdo & $\mathrm{RF}$ & RNF & Prancha & Conteúdo & RF & RNF \\
\hline A1 & Brincar & $x$ & & A12 & Pensar em fugir & & $x$ \\
\hline $\mathrm{A} 2$ & Chorar & & $x$ & A13 & Conversar & $x$ & \\
\hline A3 & Brigar & & $x$ & A14 & Ouvir música & $x$ & \\
\hline A4 & Assistir TV & $x$ & & A15 & Sentir culpa & & $x$ \\
\hline A5 & Esconder & & $x$ & A16 & Sentir medo & & $x$ \\
\hline A6 & Ficar triste & & $x$ & A17 & Ler gibi & $x$ & \\
\hline A7 & Cantar e dançar & $x$ & & A18 & Tomar remédio & $x$ & \\
\hline A8 & Rezar & $x$ & & A19 & Pensar em milagre & & $x$ \\
\hline A9 & Desanimar & & $x$ & A20 & Dormir & & $x$ \\
\hline A10 & Estudar & $x$ & & A21 & Buscar informações & $x$ & \\
\hline A11 & Fazer chantagem & & $x$ & & & & \\
\hline
\end{tabular}

Nota: RF=Respostas Facilitadoras; RNF=Respostas Não-facilitadoras. 
para me distrair) do Inventário de Estratégias de Coping, de Folkman e Lazarus (citados por Savóia et al., 1996), e no estudo realizado por Méndez et al. (1996), a partir dos quais se pode considerar tais estratégias como sendo formas de distração. Os itens 1.1. (Desenha), 1.2. (Lê ou olha revistas), 1.3. (Manuseia brinquedo) e 1.4. (Corre, pula), da Escala de Avaliação do Comportamento da Criança (Löhr \& Silvares, 1998) também contribuíram para a elaboração dessas pranchas.

Com a prancha A8: Rezar, pretendeu-se identificar se esta estratégia faz-se presente no repertório comportamental de crianças hospitalizadas com câncer e de que modo. O item 60 (Rezei), do Inventário de Estratégias de Coping, de Folkman e Lazarus, também traz esta estratégia de enfrentamento.

Na prancha A13: Conversar, pretendeu-se verificar se a criança conversa com outras crianças e sobre o quê. A proposta dessa prancha baseou-se no item 42 (Procurei um amigo ou um parente para pedir conselhos) do Inventário de Estratégias de Coping, de Folkman e Lazarus e no item 1.7 (Conversa com crianças) da Escala de Avaliação do Comportamento da Criança de Löhr e Silvares (1998). Já na prancha A21: Buscar informações, pretendeu-se saber se a criança usa como estratégia a procura de conhecimento sobre a doença, por meio de conversas com o médico ou qualquer outro profissional da equipe. O item 8 (Conversei com outra (s) pessoa (s) sobre o problema, procurando mais dados sobre a situação) do Inventário de Estratégias de Coping, de Folkman e Lazarus e o item 3.7. (Pergunta ou inicia diálogo com o médico) da Escala de Avaliação do Comportamento da Criança (Löhr \& Silvares, 1998) contribuíram para a escolha da prancha.

Com a prancha A18: Tomar remédio, pretendeu-se verificar se a criança tem disponibilidade para ser medicada, o que poderia indicar uma estratégia de enfrentamento facilitadora, indicativa de adesão ao tratamento. A Escala de Avaliação do Comportamento da Criança (Löhr \& Silvares, 1998), por meio do item 4 (Na quimioterapia ou na coleta de sangue) e do item 5 (Na medula óssea ou punção lombar), procurou identificar comportamentos de aceitação e negação do tratamento, coincidindo com a proposição da prancha A18: Tomar remédio. Itens do Inventário de Estratégias de Coping, de Folkman e Lazarus também subsidiaram a proposta desta prancha, como, por exemplo, item 49 (Eu sabia o que deveria serfeito, portanto dobrei meus esforços para fazer o que fosse necessário).

Para a prancha A2: Chorar, os itens 1.8, 2.1, 3.2, 4.3 e 5.4 (Chora) da Escala de Avaliação do Comportamento da Criança (Löhr \& Silvares, 1998), assim como as observações informais no ambiente em estudo fundamentaram sua proposição. O item 15 (Tenho vontade de chorar) da E.S.I. (Lipp \& Lucarelli, 1998) e o item 2 (Tenho vontade de chorar) da Forma C-2 do IDATE-C (Spielberg, 1983) também foram usados como referência para a elaboração da prancha.

Os itens 1.9 (Apresenta comportamento agressivo) e 1.12 (Dorme) da Escala de Avaliação do Comportamento da Criança (Löhr \& Silvares, 1998) e os itens 17 (Descontei minha raiva em outra (s) pessoa (s)) e 16 (Dormi mais que o normal) do Inventário de Estratégias de Coping, de Folkman e Lazarus contribuíram para a proposição das pranchas A3: Brigar e A20: Dormir, respectivamente. Com a prancha A3: Brigar, pretendeu-se identificar a presença de comportamentos agressivos como estratégias para enfrentar a hospitalização. No caso da prancha A20: Dormir, considerou-se que este comportamento caracteriza-se como desânimo e fuga, talvez relacionado à ociosidade, o que consistiria uma estratégia de enfrentamento nãofacilitadora.

As pranchas A5: Esconder e A12: Pensar em fugir referem-se basicamente ao mesmo tipo de estratégia, no caso, a esquiva de situações aversivas. Foram fundamentadas, indiretamente, pelo item 40 (Procurei fugir das pessoas em geral) do Inventário de Estratégias de Coping, de Folkman e Lazarus.

Os itens 10 (Eu me sinto triste) e 13 (Sinto que tenho pouca energia para fazer as coisas) da E.S.I. (Lipp \& Lucarelli, 1998) contribuíram, respectivamente, para a proposição das pranchas A6: Triste e A9: Desanimar. 
Com a prancha A11: Chantagem, pretendeu-se verificar se a criança procura usar a doença como forma de conseguir o que quer. Esse comportamento é abordado na literatura sobre doença crônica, com o nome de "ganhos secundários da doença", a partir dos quais, a pessoa se relaciona com o mundo por meio da doença (Santos \& Sebastiani, 1998).

Com a prancha A15: Culpa, pretende-se identificar sentimentos de culpa entre as crianças hospitalizadas, além de comportamentos de autopunição. O item 9 ( $M e$ (sic) critiquei, me (sic) repreendi) do Inventário de Estratégias de Coping, de Folkman e Lazarus subsidiou a proposição da prancha.

A pranchaA16: Medo teve como fundamento para a sua proposição o item 18 (Medo) do Idate-C (Spielberg, 1983) e o item 26 (Tenho medo) da E.S.I. (Lipp \& Lucarelli, 1998).

Com a prancha A19: Milagre, pretendeu-se identificar a presença do pensamento mágico na criança. $\mathrm{O}$ item 11 (Esperei que um milagre acontecesse), do Inventário de Estratégias de Coping, de Folkman e Lazarus, fundamentou sua proposição.

Por meio do Conjunto de Pranchas B: Brincar no Hospital, pretendeu-se investigar a importância atribuída ao brincar pela criança no seu processo de enfrentamento da hospitalização. Foi utilizada a classificação por família de brinquedos, proposta pelo Sistema $\mathrm{Esar}^{3}$ que permite diferenciar as expressões lúdicas, identificando os brinquedos como: jogos de exercício (E), simbólico (S), de acoplagem (A) e de regras simples e complexas (R) (Garon, 1996). Com o objetivo de ampliar as possibilidades de escolha das atividades lúdicas, foi acrescentada ao padrão de brincadeiras acima descrito uma categoria com brincadeiras que abrangiam atividades recreativas diversas (AD), como assistir à televisão, ler gibi, e contar histórias, que não puderam ser classificadas pelo Sistema Esar. O Quadro 2, a seguir, apresenta a distri-buição do brincar entre os tipos de brincadeiras selecionados.

A quantidade menor de pranchas relativas aos jogos de exercício deve-se ao fato de este tipo de brincadeira estar mais presente em idades iniciais da infância. A seqüência de apresentação foi planejada de modo a alternar as categorias.

Quarta etapa: aplicação das provas para a investigação das estratégias de enfrentamento da hospitalização da criança com câncer.

Antes da apresentação do Conjunto de Pranchas A: Enfrentamento do Hospital, a criança era questionada a respeito de seus sentimentos, pensamentos e atitudes frente à hospitalização e sua resposta, verbal, era gravada. Em seguida, foi apresentada à criança uma prancha de cada vez, devendo ela descrever a cena, para, em seguida, responder o quanto aquela figura se parecia com o que ela vinha fazendo durante o tempo que passava no hospital.

Para registrar a resposta, foram oferecidos à criança cinco círculos de velcro, de tamanho e cores iguais, que deveriam ser fixados no círculo preso ao caderno de respostas. Dessa forma, a criança deveria fixar um círculo quando achasse

Quadro 2. Conjunto de Pranchas B: Brincar no Hospital.

\begin{tabular}{ll}
\hline Jogos de Exercício & Bola; Tocar \\
Jogos Simbólicos & Fantoches; Palhaço; Desenhar; Médico \\
Jogos de Acoplagem & Montagem; Modelagem; Recorte/colagem; Quebra-cabeça \\
Jogos de Regras & Baralho; Minigame; Dominó; Bingo; Dama \\
Atividades Diversas & Assistir TV; Ler gibi; Ouvir histórias; Vários brinquedos; Cantar e dançar \\
\hline
\end{tabular}

\footnotetext{
${ }^{3}$ Esar: Sistema de classificação de jogos e brinquedos, criado por Denise Garon, psicopedagoga pré-escolar, analista de jogos e brinquedos, e professora do Collège Professionel de Saint-Foy, Quebec, Canadá (Garon, 1996).
} 
que havia feito apenas às vezes o que estava na figura; dois círculos no caso de quase sempre, três círculos no caso de sempre, e nenhum para o caso de nunca ter feito.

Após a escolha de cada figura, a criança era questionada sobre o motivo de sua resposta, que foi gravada. As respostas às figuras foram, posteriormente, anotadas também na folha de registro pela pesquisadora.

Para o Conjunto de Pranchas B: Brincar no Hospital, foi mantido o mesmo processo desenvolvido no Conjunto de Pranchas A.

\section{RESULTADOS}

Considerando os objetivos deste artigo apresentar uma proposta de avaliação das estratégias de enfrentamento da hospitalização em crianças com câncer, serão descritos somente os resultados obtidos a partir do Conjunto A: Enfrentamento da Hospitalização, por ser esta a parte do instrumento que permite obter dados diretos sobre as estratégias de enfrentamento da hospitalização ${ }^{4}$.

Com o objetivo de verificar se os desenhos estavam coerentes com o que se desejava transmitir, foi realizada uma análise da adequação das pranchas a partir da descrição da criança. Para tanto, foram criadas as seguintes categorias: a) adequada sem ajuda (A): a criança descrevia e nomeava a prancha corretamente sem a ajuda da pesquisadora; b) adequada com ajuda (Aa): resposta adequadamente dada pela criança com a ajuda da pesquisadora que, neste caso, fazia perguntas a fim de direcionar e facilitar a compreensão da criança sobre o tema abordado pela prancha e c) inadequada (I): incluíram-se aqui aquelas respostas muito diferentes da esperada para a prancha.

De um modo geral, os resultados referentes à adequação das pranchas do Conjunto $A$ : Enfrentamento da Hospitalização mostraram que $88,4 \%$ das descrições das pranchas, feitas pelas crianças, foram consideradas adequadas, indicando necessidade de revisão das pranchas "Ficar triste" e "Sentir medo", uma vez que as descrições recebidas não atingiram o percentual mínimo de 80,0\% de adequação. As pranchas "Esconder" e "Sentir culpa" atingiram 80,0\% de adequação após passarem por alterações em seu desenho, a partir da $11^{\mathrm{a}}$ e $14^{\mathrm{a}}$ criança entrevistadas, respectivamente.

É apresentada a adequação das pranchas do AEH a partir da descrição feita pelas crianças hospitalizadas com câncer (Tabela 1).

O grupo de pranchas representativas de estratégias facilitadoras apresentou um percentual de adequação superior (94,6\%) àquele observado no grupo de pranchas representativas de estratégias não-facilitadoras (82,8\%). De fato, as cenas das pranchas sobre estratégias facilitadoras caracterizam-se por conteúdos mais simples de serem representados graficamente,

Tabela 1. Adequação das respostas das crianças às pranchas do $\mathrm{AEH}$.

\begin{tabular}{|c|c|c|c|c|c|}
\hline \multirow{2}{*}{ AEH Respostas } & & \multicolumn{2}{|c|}{ Adequada (\%) } & \multicolumn{2}{|c|}{ Subtotal (\%) } \\
\hline & & Sem ajuda & Com ajuda & Adequada & Inadequada \\
\hline Conjunto A: & Estratégias & 85,0 & 9,6 & 94,6 & 5,4 \\
\hline Enfrentamento da & Facilitadoras & & & & \\
\hline Hospitalização & Estratégias & 57,5 & 25,3 & 82,8 & 17,2 \\
\hline & Não-facilitadoras & & & & \\
\hline
\end{tabular}

\footnotetext{
${ }^{4}$ O Conjunto B: Brincar no Hospital é específico para a investigação do uso do brincar como estratégia de enfrentamento da hospitalização e já foi descrito em publicação anterior (Motta \& Enumo, 2002).
} 
por se referirem mais a ações do que a sentimentos ou pensamentos. Além disso, pode-se pensar que perceber situações mais prazerosas pode ser mais fácil do que descrever situações de conflito ou desprazer.

\section{Respostas das crianças hospitalizadas com câncer às pranchas do $\mathrm{AEH}$}

Os resultados relativos às respostas das crianças nas pranchas do Conjunto A: Enfrentamento da Hospitalização indicam um percentual de $53,2 \%$ de respostas sim (às vezes, quase sempre e sempre) e $46,8 \%$ de respostas não. As pranchas que receberam o maior número de respostas sim foram, em sua maioria, aquelas representativas de "estratégias de enfrentamento facilitadoras": brincar (A1: 92,9\%), assistir TV (A4: 89,3\%), rezar (A8: 82,1\%) e tomar remédio (A18: 92,9\%).

As pranchas que receberam o maior número de respostas não foram: A3: Brigar (100\%), A5: Esconder (92,9\%), A12: Pensar em fugir $(96,4 \%) \mathrm{e}$ A15: Sentir culpa (82,1\%). As pranchas A2: Chorar, A6: Ficar triste, A16: Sentir medo, A9: Desanimar, A19: Pensar em milagre e A20: Dormir, representativas de "estratégias não-facilitadoras" de enfrentamento da hospitalização, receberam um número de respostas sim superior ao de respostas não.

Para a análise das justificativas das respostas dadas pelas crianças nas pranchas, foi realizada uma categorização, cujo processo será descrito a seguir.

Utilizando-se o referencial da análise funcional do comportamento ${ }^{5}$ (Skinner, 1978), considerou-se que cada comportamento avaliado poderia acontecer em função de situações antecedentes que o desencadeavam e/ou em função das conseqüências que o mesmo poderia trazer para a criança, que poderia justificar suas respostas afirmativas e negativas. Assim, foram criadas as categorias descritas a seguir.

\section{Categorias fundamentadas em eventos antecedentes à resposta analisada:}

1.1 Ambiente hospitalar: recursos materiais, pessoas, local específico, rotina do hospital e ociosidade justificavam tanto a ocorrência quanto a não ocorrência do comportamento.

\subsection{Contexto da doença e do tratamento:} os efeitos da medicação, a adaptação à doença, a avaliação positiva do quadro clínico, os procedimentos invasivos, a depressão, a duração e/ou persistência da doença, o prognóstico da doença, a preocupação com o tratamento, a rotina do tratamento justificavam tanto a ocorrência quanto a não ocorrência da resposta.

1.3 Característica da criança: características pessoais, crenças, valores e regras da criança justificam a ocorrência e a não ocorrência da resposta.

1.4 Contexto familiar: quando atitudes dos familiares, experiências vividas em casa e restrições à convivência diária com parentes e amigos, ao brincar e a outros aspectos do seu cotidiano justificavam tanto a ocorrência quanto a não ocorrência da resposta.

\section{Categorias fundamentadas em eventos conseqüentes à resposta analisada:}

2.1 Conseqüências positivas: sensações e sentimentos positivos, aprendizagem, controle da situação, diminuição e eliminação da aversividade da situação, conhecimento geral sobre a doença, passar de ano, ganhar o que quer, ter companhia e a atividade por si só.

2.2 Conseqüências negativas: sensações e sentimentos negativos, reprovação social, mal-estar físico, aumento da aversividade da situação, exposição aos outros e conseqüências negativas para si próprio e para os outros.

Os resultados indicaram que as escolhas ou não das pranchas relativas a "estratégias de

\footnotetext{
${ }^{5}$ De um modo geral, a análise funcional é usada para compreender o comportamento de um indivíduo. Nesta perspectiva, existe um comportamento, considerado uma variável dependente, para o qual se investigam as causas. Estas, por sua vez, chamadas variáveis independentes, referem-se às condições externas das quais o comportamento é função (Skinner, 1978).
} 
enfrentamento da hospitalização facilitadoras" decorreram tanto de eventos antecedentes, relacionados principalmente ao ambiente hospitalar, quanto de eventos conseqüentes relacionados às conseqüências positivas proporcionadas.

No que se refere às escolhas ou não das pranchas representativas de "estratégias não-facilitadoras" de enfrentamento da hospitalização, viu-se que estas se deveram tanto a eventos antecedentes relacionados principalmente ao contexto da doença e do tratamento quanto a eventos conseqüentes. Verificou-se que os procedimentos médicos invasivos, como punções, exame de sangue e injeções, foram freqüentemente indicados como geradores de "estratégias de enfrentamento não-facilitadoras", sendo comuns relatos como: "Só choro quando vai pegar minha veia. (Q) Porque tem vez que fica furando um montão de vez, aí, eu choro" (C24, F, 9 anos).

\section{DISCUSSÃO}

Esta pesquisa pretende apresentar uma proposta de elaboração de um instrumento para a avaliação das estratégias utilizadas por crianças com câncer para enfrentar a hospitalização.

Considerando que as estratégias de enfrentamento dizem respeito aos comportamentos e pensamentos das pessoas ao lidarem com situações estressantes, é possível acessá-las através do inquérito verbal, como propõem os autores de escalas e inventários (Lazarus \& Folkman, 1984; Lipp \& Lucarelli, 1998; para citar alguns). Entretanto, há situações em que o uso exclusivo da técnica da entrevista pode não ser suficiente ou adequado, como ocorre com a condição de hospitalização, em que a criança encontra-se em um local incomum a sua rotina e vivenciando situações de difícil manejo. Havia, assim, um desafio metodológico a ser enfrentado: como garantir a participação de crianças hospitalizadas, com uma doença grave, que exige internações e procedimentos dolorosos, de forma a levá-las a falar justamente sobre essa condição aversiva, a hospitalização? E por que queríamos fazer isso?

A solução para a questão metodológica foi o resgate de um recurso antigo da Psicologia - 0 desenho $0^{6}$ - como instrumento de coleta de dados; dada a sua proximidade com o universo infantil, da mesma maneira que o brincar.

Propor pranchas com desenhos ampliou as possibilidades de expressão dos sentimentos, comportamentos e pensamentos das crianças com câncer sobre a hospitalização, o que foi constatado frente à diferença de conteúdos obtidos por meio das questões abertas e das questões intermediadas pelas pranchas. Estas últimas serviram como potenciais facilitadores para a identificação e compreensão psicológica da criança com câncer hospitalizada, podendo assim fornecer subsídios para intervenções psicológicas e ações institucionais consistentes.

Em se tratando de um recurso para auxiliar a equipe de saúde mental, o material proposto pode subsidiar as técnicas de modificação de conduta a serem instauradas, no sentido de tornar mais positivas e adequadas as estratégias de enfrentamento utilizadas pelas crianças hospitalizadas. Assim, quando se identificam na criança respostas indicativas de estratégias não-facilitadoras, como desânimo, medo, tristeza e choro frente à exposição a procedimentos médicos, podem ser incorporadas ao tratamento psicológico da criança hospitalizada, técnicas que facilitam o enfrentamento adequado, como, por exemplo, o relaxamento e o controle da respiração, a imaginação e a distração, e o ensaio de conduta, entre outras, citadas no estudo sobre a preparação da hospitalização infantil de Méndez et al. (1996).

O instrumento proposto não é apenas uma escala que mede valores numéricos do comportamento, uma vez que usa o inquérito para aprofundar as respostas. Este é um diferencial, juntamente com o estímulo do desenho para as perguntas a serem feitas para a criança,

\footnotetext{
${ }^{6}$ Em 1954, foi publicado um número especial do Boletim de Psicologia sobre o I Ciclo de Estudos sobre o Desenho, pela Sociedade de Psicologia de São Paulo (Fávero \& Salim, 1995).
} 
e também a forma de registro oferecida, que se assemelha a um jogo. O caráter lúdico do material mostrou ser capaz de envolver e motivar a participação da criança.

Por fim, considere-se a possibilidade de que esta pesquisa se caracterize principalmente como um estudo-piloto para a realização de um trabalho fundamentalmente metodológico, planejado para ser submetido a análises estatísticas consistentes, a fim de ser validado como um instrumento capaz de contribuir para o diagnóstico e para a intervenção junto à criança hospitalizada.

\section{AGRADECIMENTOS}

Ao CNPq, cuja bolsa permitiu a realização da Dissertação de Mestrado - Brincar no hospital: Câncer infantil e avaliação do enfrentamento da hospitalização. Programa de Pós-Graduação em Psicologia, Universidade Federal do Espírito Santo - defendida pela primeira autora, orientada pela segunda autora.

\section{REFERÊNCIAS}

Antoniazzi, A.S., Dell'aglio, D.D., \& Bandeira, D.R. (1998). O conceito de coping: Uma revisão teórica. Estudos de Psicologia, 3 (2), 273-294.

Assumpção, F., Kuczynski, E., Sprovieri, M.H., \& Aranha, E.M.G. (2000). Escala de avaliação de qualidade de vida: Validade e confiabilidade de uma Escala para Qualidade de Vida em crianças de 4 a 12 anos. Arquivos de Neuro-Psiquiatria, 58 (1), 119-127.

Cerqueira, A.T.A.R. (2000). O conceito e metodologia de coping: Existe consenso e necessidade? In R.R. Kerbauy (Org.). Sobre Comportamento e cognição: Psicologia Comportamental e Cognitiva- conceitos, pesquisa e aplicação, a ênfase no ensinar, na inovação e no questionamento clínico (v.5, p.279-289). Santo André: Arbytes Editora.

Fávero, M.H., \& Salim, C.M.R. (1995). A relação entre os conceitos de saúde, doença e morte: Utilização do desenho na coleta de dados. Psicologia: Teoria e Pesquisa, 11 (3), 181-191.
Garon, D. (1996). Classificação e análise de materiais lúdicos: O Sistema ESAR. In A. Friedmann, C. Aflalo, C.M.R.J. Andrade \& R.Z. Altman (Orgs.). O Direito de Brincar (p. 173-186). São Paulo: Scritta: Abrinq.

Lazarus, R., \& Folkman, S. (1984). Stress, appraisal, and coping. New York: Springer Publishing Company.

Lipp, M.E.N., \& Lucarelli, M.D.M. (1998). Manual da Escala de Stress Infantil - ESI. São Paulo: Casa do Psicólogo.

Löhr, S. (1998). Crianças com câncer: Discutindo a intervenção psicológica. Tese de Doutorado não publicada, Universidade de São Paulo.

Löhr, S., \& Silvares, E.F.M. (1998). Escala de avaliação do comportamento da criança. In S.S. Lohr. Crianças com câncer: Discutindo a intervenção psicológica (p.136-138). Tese de Doutorado não publicada, Universidade de São Paulo.

Méndez, F.X., Ortigosa, J.M., \& Pedroche, S. (1996). Preparación a la hospitalización infantil (I): Afrontamiento del estrés. Psicologia Conductual, 4 (2), 193-209.

Motta, A.B., Enumo, S.R.F. (2002). Brincar no hospital: Câncer infantil e avaliação do enfrentamento da hospitalização. Psicologia: Saúde e Doenças, 3 (1), 23-41.

Santos, C.T., \& Sebastiani, R.W. (1998). Acompanhamento psicológico à pessoa portadora de doença crônica. In V.A. Angerami-Camon. E a Psicologia entrou no hospital (p.147-176). São Paulo: Pioneira.

Savóia, M.G., Santana, P.R., \& Mejias, N.P. (1996). Adaptação do Inventário de Estratégias de Coping de Folkman e Lazarus para o Português. Psicologia USP, 7 (1/2), 183-201.

Skinner, B.F. (1978). Ciência e comportamento humano (4.ed.). São Paulo: Martins Fontes.

Spielberg, C.D. (1983). Manual do Inventário de Ansiedade Traço-Estado - IDATE-C. Rio de Janeiro: CEPA.

Recebido para publicação em 23 de abril e aceito em 17 de novembro de 2004. 